\title{
An Alternative Converse Lyapunov Theorem for Discrete-Time Systems
}

\author{
Roman Geiselhart*1, Rob H. Gielen ${ }^{2}$, Mircea Lazar², and Fabian R. Wirth ${ }^{1}$ \\ ${ }^{1}$ Institute for Mathematics, University of Würzburg, Germany \\ ${ }^{2}$ Department of Electrical Engineering, Eindhoven University of Technology, The Netherlands
}

June 14, 2013

\begin{abstract}
This paper presents an alternative approach for obtaining a converse Lyapunov theorem for discrete-time systems. The proposed approach is constructive, as it provides an explicit Lyapunov function. The developed converse theorem establishes existence of global Lyapunov functions for globally exponentially stable (GES) systems and semi-global practical Lyapunov functions for globally asymptotically stable systems. Furthermore, for specific classes of systems, the developed converse theorem can be used to establish non-conservatism of a particular type of Lyapunov functions. Most notably, a proof that conewise linear Lyapunov functions are non-conservative for GES conewise linear systems is given and, as a by-product, tractable construction of polyhedral Lyapunov functions for linear systems is attained.
\end{abstract}

Key Words. Discrete-time systems, Conewise linear systems, Stability analysis, Converse Lyapunov theorems

\section{Introduction}

Lyapunov functions are a powerful tool for establishing stability properties of dynamical systems, such as global asymptotic stability (GAS). The existence of a Lyapunov function is guaranteed by converse Lyapunov theorems under the assumption that the system is GAS, see e.g., $[1,2,3,4,5,6$, $7,8,9]$, and the references therein. For the discrete-time case, which is of interest in this work, the abstract construction of a global Lyapunov function for a converse theorem is performed by taking infinite sums [3] or the supremum over all solutions and all times $[8,5]$. As such, these approaches require the knowledge of solutions for all positive times. For certain classes of dynamics there are classes of Lyapunov functions that preserve necessity, such as quadratic functions [4] for linear difference equations and polyhedral functions $[1,10,11]$ for linear difference inclusions. However, for the case of polyhedral Lyapunov functions, developing tractable constructive methods is still an open problem.

Existing results on constructive converse Lyapunov theorems for general nonlinear systems are scarce and come with certain limitations, as discussed next. In the monograph [12] converse Lyapunov theorems for continuous-time systems are obtained via piecewise linear Lyapunov functions and linear programming. Another relevant result for continuous-time systems can be found in [13], where the authors show the relation between control Lyapunov functions and solutions to generalized Zubov equations, i.e., a first order partial differential equation. A result relevant for discrete-time systems was given in [14], where it was shown that for a globally exponentially stable (GES) discrete-time system a Lyapunov function can be constructed by a finite sum of solutions. This was established under the assumption that the system dynamics is continuous and locally Lipschitz.

\footnotetext{
${ }^{*}$ Corresponding author;

Emails: \{roman.geiselhart,wirth\}@mathematik.uni-wuerzburg.de,r.h.gielen@hotmail.com,m.lazar@tue.nl
} 
In this paper, an alternative approach to construction of Lyapunov functions for discrete-time systems is proposed. Continuity of the systems dynamics is not required and the proposed approach is shown to hold for a larger class of systems than GES systems.

The first ingredient of the proposed approach consists of a relaxation of the Lyapunov function concept, which was originally introduced in [15]: the Lyapunov function is required to decrease along the system solutions after a finite number of time steps, and not at every time step. It is shown that this relaxation, termed finite-time Lyapunov function in this work, still yields sufficient conditions for establishing GAS of the underlying system, without requiring continuity of the system dynamics. Secondly, a converse finite-time Lyapunov theorem is derived. This converse theorem is constructive in that it yields a global finite-time Lyapunov function that is explicitly given. Then, a way to construct a standard Lyapunov function based on the knowledge of a finitetime Lyapunov function and a corresponding natural number is given. The construction depends only on a finite sum and hence, it is straightforward to implement.

Combining the above results yields the first main contribution of this paper, i.e., a novel converse Lyapunov theorem that provides an explicit construction of a Lyapunov function for a large class of discrete-time systems. More specifically, global Lyapunov functions for GES discrete-time systems, and (at least) semi-global practical Lyapunov functions for GAS discrete-time systems can be constructed. The Lyapunov function construction hinges of finding a suitable natural number a priori. Several possibilities to find such a suitable number in a systematic way are discussed for certain classes of systems. Of course, finding such a suitable number may be undecidable or computationally intractable, as it is well known that stability analysis and hence, also construction of Lyapunov functions, is an NP-hard problem in general [16].

The second main contribution of this paper is to establish non-conservatism of specific types of Lyapunov functions via the developed converse theorem. Most notably, it is established that conewise linear Lyapunov functions are non-conservative for GES conewise linear systems, which is one of the open problems in stability analysis of hybrid systems [17]. The latter result further yields, as a by-product, a new method to construct polyhedral Lyapunov functions for linear systems, which is tractable even in state spaces of high dimension.

The remainder of this paper is structured as follows. The necessary preliminaries are given in Section 2 and the definition of global (finite-time) Lyapunov functions as well as the problem statement are given in Section 3. Section 4 contains the main results of this paper. In Section 4.1 global finite-time Lyapunov functions are shown to be sufficient for the system being GAS, whereas in Section 4.2 also necessity is proven under an appropriate assumption. In Section 4.3 it is shown how to construct a global Lyapunov function from the knowledge of a global finite-time Lyapunov function, which combined with the results from Section 4.2 gives a converse Lyapunov theorem in Section 4.4. Relevant implications of the developed converse theorem are presented in Section 5, while conclusions are drawn in Section 6.

\section{Preliminaries}

By $\mathbb{N}$ we denote the natural numbers and we assume $0 \in \mathbb{N}$. Let $\mathbb{R}$ denote the field of real numbers, $\mathbb{R}_{+}$the set of nonnegative real numbers and $\mathbb{R}^{n}$ the vector space of real column vectors of length $n$. For a vector $x \in \mathbb{R}^{n}$ we denote by $[x]_{i}$ its $i$-th entry. Accordingly, for a matrix $A \in \mathbb{R}^{n \times m}$ we denote by $[A]_{i, j}$ its $(i, j)$-th entry. Furthermore, the notation $[A]_{i,:}$ denotes the $i$-th row (respectively, $[A]_{:, j}$ denotes the $j$-th column) of matrix $A$. For matrices $A_{1}, \ldots, A_{N} \in \mathbb{R}^{n \times m}$ we use the abbreviation $\left(A_{1} ; \ldots ; A_{N}\right):=\left(A_{1}^{\top} \ldots A_{N}^{\top}\right)^{\top}$.

By $\|\cdot\|$ we denote any arbitrary norm on $\mathbb{R}^{n}$. In particular, we use the 1-norm $\|x\|_{1}=\sum_{i=1}^{n}\left|[x]_{i}\right|$ and the infinity norm $\|x\|_{\infty}=\max _{i \in\{1, \ldots, n\}}\left|[x]_{i}\right|$. To state the stability results, we use standard comparison functions. We call a function $\alpha: \mathbb{R}_{+} \rightarrow \mathbb{R}_{+}$a function of class $\mathcal{K}$ (denoted by $\alpha \in \mathcal{K}$ ), if it is strictly increasing, continuous, and satisfies $\alpha(0)=0$. In particular, if $\alpha \in \mathcal{K}$ is unbounded, it is said to be of class $\mathcal{K}_{\infty}$. A function $\beta: \mathbb{R}_{+} \times \mathbb{R}_{+} \rightarrow \mathbb{R}_{+}$is called a function of class $\mathcal{K} \mathcal{L}(\beta \in \mathcal{K} \mathcal{L})$, if it is of class $\mathcal{K}$ in the first argument and strictly decreasing to zero in the second argument. A 
function $\eta: \mathbb{R}_{+} \rightarrow \mathbb{R}_{+}$is called positive (semi-)definite, if $\eta(0)=0$ and $\eta(s)>0$ (resp. $\eta(s) \geq 0$ ) for all $s>0$. For two functions $\alpha_{1}, \alpha_{2} \in \mathcal{K}_{\infty}$, we denote $\alpha_{1}<\alpha_{2}$ (resp. $\alpha_{1} \leq \alpha_{2}$ ) if $\alpha_{2}-\alpha_{1}$ is positive (semi-)definite. Furthermore, $\alpha_{1} \circ \alpha_{2}$ denotes the composition, and $\alpha_{1}^{k}:=\alpha_{1} \circ \ldots \circ \alpha_{1}$ is the $k$-th iterate of $\alpha_{1}$. For $\rho \in \mathcal{K}_{\infty}, j \in \mathbb{Z}, M \in \mathbb{N}, M>0$, we denote by $\gamma:=\rho^{j / M}$ a solution of the equation $\gamma^{M}=\rho^{j}$, that exists by [18, Proposition 3.1]. A function $G: \mathbb{R}^{n} \rightarrow \mathbb{R}^{n}$ is called $\mathcal{K}$-bounded, if there exists an $\omega \in \mathcal{K}$ such that

$$
\|G(x)\| \leq \omega(\|x\|), \quad \forall x \in \mathbb{R}^{n} .
$$

\section{Problem statement}

We consider discrete-time systems of the form

$$
x(k+1)=G(x(k)), \quad k \in \mathbb{N},
$$

where $G: \mathbb{R}^{n} \rightarrow \mathbb{R}^{n}$ is assumed to satisfy the following standing assumption.

Assumption 1. The function $G$ in (1) is $\mathcal{K}$-bounded.

Compared to the typical assumptions employed in converse Lyapunov theorems, Assumption 1 is not restrictive, as it does not require continuity of the map $G(\cdot)$ (except ${ }^{1}$ at $x=0$ ). On the other hand, any continuous map $G: \mathbb{R}^{n} \rightarrow \mathbb{R}^{n}$ with $G(0)=0$ is $\mathcal{K}$-bounded. Also, for any GES system (1) the map $G(\cdot)$ is $\mathcal{K}$-bounded with a linear function $\omega$, as worked out further on in this section.

By $x(k, \xi) \in \mathbb{R}^{n}$ we denote the solution of system (1) at time instance $k \in \mathbb{N}$ with initial condition $x(0)=\xi \in \mathbb{R}^{n}$.

Definition 2. The system (1) is called globally asymptotically stable (GAS) if there exists a $\mathcal{K} \mathcal{L}$ function $\beta$ such that for all $\xi \in \mathbb{R}^{n}$ and all $k \in \mathbb{N}$

$$
\|x(k, \xi)\| \leq \beta(\|\xi\|, k) .
$$

If the $\mathcal{K} \mathcal{L}$-function in $(2)$ can be chosen as

$$
\beta(r, t)=C \mu^{t} r
$$

with $C \geq 1$ and $\mu \in[0,1)$, then system (1) is called globally exponentially stable (GES).

Remark 3. The definition of GES is somehow misleading, since global only indicates that (2) with $\beta$ as in (3) holds for all $\xi \in \mathbb{R}^{n}$. Since $C \geq 1$ in (3) is chosen globally, it does not reflect the local behavior of a particular solution near 0 . Note that this property is also often called exponentially stable in the large, see e.g., [2].

Note that for a GES system the $\mathcal{K}$-bound $\omega$ on $G$ in (1) can always be chosen to be linear. This follows directly, since $\|G(\xi)\|=\|x(1, \xi)\| \leq C \mu^{1}\|\xi\|$ for all $\xi \in \mathbb{R}^{n}$.

Definition 4. A function $W: \mathbb{R}^{n} \rightarrow \mathbb{R}_{+}$is a global Lyapunov function for system (1) if

(i) there exist $\alpha_{1}, \alpha_{2} \in \mathcal{K}_{\infty}$ such that for all $\xi \in \mathbb{R}^{n}$

$$
\alpha_{1}(\|\xi\|) \leq W(\xi) \leq \alpha_{2}(\|\xi\|),
$$

(ii) there exists a $\mathcal{K}$-function $\rho$ satisfying $\rho<$ id such that for all $\xi \in \mathbb{R}^{n}$

$$
W(x(1, \xi)) \leq \rho(W(\xi)) .
$$

\footnotetext{
${ }^{1}$ Recall that continuity at $x=0$ is a necessary condition for Lyapunov stability of the origin.
} 
Remark 5. In many prior works (e.g., [3]) the definition of a Lyapunov function requires the existence of a positive definite function $\alpha_{3}$ such that $W(x(1, \xi))-W(\xi) \leq-\alpha_{3}(\|\xi\|)$ holds for all $\xi \in \mathbb{R}^{n}$. Let us briefly explain, that this requirement is equivalent to Definition 4 . Note that by following similar steps as in [19, Theorem 2.3.5] we conclude

$$
\begin{aligned}
W(x(1, \xi)) & \leq W(\xi)-\alpha_{3}(\|\xi\|) \\
& \leq\left(\mathrm{id}-\alpha_{3} \circ \alpha_{2}^{-1}\right)(W(\xi))=\rho(W(\xi))
\end{aligned}
$$

with $\rho:=\left(\right.$ id $\left.-\alpha_{3} \circ \alpha_{2}^{-1}\right)$ positive definite. We further have $0 \leq W(x(1, \xi)) \leq\left(\alpha_{2}-\alpha_{3}\right)(\|\xi\|)$, so $\alpha_{2} \geq \alpha_{3}$ and therefore $\rho<\mathrm{id}$. Without loss of generality we can assume that $\rho \in \mathcal{K}$. On the other hand for a given $\rho<$ id we obtain $W(x(1, \xi))-W(\xi) \leq-\alpha_{3}(\|\xi\|)$ for $\alpha_{3}:=($ id $-\rho) \circ \alpha_{1}$. Furthermore, for the case $\alpha_{1}(s)=a s^{\lambda}, \alpha_{2}(s)=b s^{\lambda}, \alpha_{3}(s)=c s^{\lambda}$ for some $a, b, c, \lambda>0$ we have $W(x(1, \xi)) \leq \rho W(\xi)$ with $\rho:=\left(1-\frac{c}{b}\right) \in[0,1)$, see [19, Theorem 2.3.5].

Next, the assumptions on the global Lyapunov function given in Definition 4 is relaxed as follows.

Definition 6. A function $V: \mathbb{R}^{n} \rightarrow \mathbb{R}_{+}$is a global finite-time Lyapunov function for system (1) if

(i) there exist $\alpha_{1}, \alpha_{2} \in \mathcal{K}_{\infty}$ such that for all $\xi \in \mathbb{R}^{n}$

$$
\alpha_{1}(\|\xi\|) \leq V(\xi) \leq \alpha_{2}(\|\xi\|)
$$

(ii) there exists a finite $M \in \mathbb{N}$ and a $\mathcal{K}$-function $\rho<$ id such that for all $\xi \in \mathbb{R}^{n}$

$$
V(x(M, \xi)) \leq \rho(V(\xi)) .
$$

It is worth pointing out that the concept of a global finite-time Lyapunov function was originally introduced in [15], which dealt with stability analysis of time-varying systems, although the term finite-time was not used therein. Clearly, any global Lyapunov function is a particular global finite-time Lyapunov function.

\section{Main results}

The aim of this section is to derive a constructive converse Lyapunov theorem for systems of the form (1). To this aim we proceed by showing that any global finite-time Lyapunov function guarantees GAS of the underlying dynamical system. We show that the converse, i.e,. the existence of a global finite-time Lyapunov function for a GAS system, also holds and, moreover, that under an appropriate assumption the existence is guaranteed for any scaled norm as a global finite-time Lyapunov function. In particular, we show that the assumption is satisfied for any GES system of the form (1). We further prove that any scaled norm is at least a semi-global practical finite-time Lyapunov function (see Definition 17) for a GAS system of the form (1). To obtain a converse Lyapunov theorem, an explicit construction of a global Lyapunov function from a global finite-time Lyapunov function is provided.

\subsection{Finite-time Lyapunov functions}

This section proceeds by showing that the existence of a global finite-time Lyapunov function is sufficient to conclude GAS of the underlying system.

Theorem 7. The existence of a global finite-time Lyapunov function implies that system (1) is GAS.

Proof. Assume that there exists a global finite-time Lyapunov function $V$ as defined in Definition 6 . First note that from the standing Assumption 1 we conclude that for any $j \in \mathbb{N}$

$$
\|x(j, \xi)\| \leq \omega^{j}(\|\xi\|) .
$$


Then for any $k=l M+j, l \in \mathbb{N}, j \in\{0, \ldots, M-1\}$ we have

$$
\begin{aligned}
\|x(k, \xi)\| & \leq \alpha_{1}^{-1}(V(x(k, \xi))) \\
& \leq \alpha_{1}^{-1}(V(x(l M, x(j, \xi)))) \\
& \leq \alpha_{1}^{-1} \circ \rho^{l}(V(x(j, \xi))) \\
& \leq \alpha_{1}^{-1} \circ \rho^{l} \circ \alpha_{2}(\|x(j, \xi)\|) \\
& \stackrel{(4)}{\leq} \alpha_{1}^{-1} \circ \rho^{l} \circ \alpha_{2} \circ \omega^{j}(\|\xi\|) \\
& \leq \max _{i \in\{0, \ldots, M-1\}} \alpha_{1}^{-1} \circ \rho^{l} \circ \alpha_{2} \circ \omega^{i}(\|\xi\|) \\
& \leq \max _{i \in\{0, \ldots, M-1\}} \alpha_{1}^{-1} \circ \rho^{\frac{k}{M}-1} \circ \alpha_{2} \circ \omega^{i}(\|\xi\|) \\
& =: \beta(\|\xi\|, k) .
\end{aligned}
$$

It is easy to see that $\beta$ is a $\mathcal{K} \mathcal{L}$-function. Then by Definition 2 system (1) is GAS.

If the global finite-time Lyapunov function satisfies some conditions on the functions invoked, then we can conclude GES.

Corollary 8. Let the $\mathcal{K}$-bound on $G$ be $\omega(s)=w s$ for all $s \geq 0$ and $w>0$. Then the existence of a global finite-time Lyapunov function $V$ satisfying Definition 6 with

$$
\alpha_{1}(s)=a_{1} s^{\lambda}, \alpha_{2}(s)=a_{2} s^{\lambda}, \rho(s)=c s
$$

with $0<a_{1} \leq a_{2}, \lambda>0$ and $c \in[0,1)$ implies that system (1) is GES.

Proof. The proof of Theorem 7 implies $\|x(k, \xi)\| \leq C \mu^{k}\|\xi\|$ with $C=\max _{i \in\{1, \ldots, M-1\}}\left(\frac{a_{2}}{a_{1} c}\right)^{1 / \lambda} \omega^{i}$ and $\mu=c^{1 / M \lambda} \in[0,1)$.

Note that the assumption on $\omega$ to be linear is necessary for the system being GES as previously indicated.

Since any global finite-time Lyapunov function with $M=1$ is even a global Lyapunov function, Lyapunov's theorem can also be obtained as a corollary of Theorem 7 .

Corollary 9. The existence of a global Lyapunov function implies that system (1) is GAS.

The same holds true for the GES case by means of Corollary 8.

The following result establishes that if the map $G$ is a contraction, then system (1) is GAS.

Proposition 10. If the map $G$ in $(1)$ is $\mathcal{K}$-bounded with $\mathcal{K}$-function $\omega<\mathrm{id}$, then system (1) is GAS.

Proof. Take $W(\xi)=\|\xi\|$ as a candidate Lyapunov function. Then clearly Definition $4-(\mathrm{i})$ is satisfied with $\alpha_{1}=\alpha_{2}=$ id and Definition $4-$ (ii) is satisfied with $\rho=\omega$ and $\omega<$ id. So Corollary 9 applies, which concludes the proof.

\subsection{A converse finite-time Lyapunov function theorem}

This section proceeds by showing that the existence of a global finite-time Lyapunov function is also necessary.

Proposition 11. If system (1) is GAS, then there exists a global finite-time Lyapunov function. 
Proof. Any global Lyapunov function as defined in Definition 4 is a global finite-time Lyapunov function with $M=1$. So with [3, Theorem 1] GAS together with a continuous dynamic map $G(\cdot)$ implies the existence of a global finite-time Lyapunov function. Note that in [3] the authors take the Euclidian norm and define stability with respect to a set $\mathcal{A}$. Since the proof holds by taking any arbitrary norm this result holds with $\mathcal{A}=\{0\}$. See also [8] for the case when $G$ is discontinuous.

Proposition 11 ensures the existence of a global finite-time Lyapunov function by using a standard converse Lyapunov theorem, which is obvious. Indeed, a converse result was not pursued in $[15]$.

Next, a constructive converse finite-time Lyapunov theorem is stated, which does not rely on a standard Lyapunov function, but on an appropriate assumption that is discussed in the following.

Assumption 12. There exists a $\mathcal{K} \mathcal{L}$-function $\beta$ satisfying (2) for system (1) and

$$
\beta(r, M)<r
$$

for some $M \in \mathbb{N}$ and all $r>0$.

Under this assumption a global finite-time Lyapunov function can be given explicitly.

Theorem 13. If Assumption 12 is satisfied, then for any function $\eta \in \mathcal{K}_{\infty}$ the function $V: \mathbb{R}^{n} \rightarrow$ $\mathbb{R}_{+}$defined by

$$
V(\xi):=\eta(\|\xi\|)
$$

for all $\xi \in \mathbb{R}^{n}$ is a global finite-time Lyapunov function for system (1).

Proof. Take any $\eta \in \mathcal{K}_{\infty}$ and let $V$ be defined as in (7). Then clearly Definition 6-(i) is satisfied. Let $\beta \in \mathcal{K} \mathcal{L}$ satisfy (2), and $M \in \mathbb{N}$ satisfy (6). Then for any $\xi \in \mathbb{R}^{n}$

$$
\begin{aligned}
V(x(M, \xi)) & =\eta(\|x(M, \xi)\|) \\
& \leq \eta \circ \beta(\|\xi\|, M) \\
& =\eta \circ \beta\left(\eta^{-1}(V(\xi)), M\right) \\
& =\tilde{\rho}(V(\xi)),
\end{aligned}
$$

where $\tilde{\rho}(\cdot):=\eta \circ \beta\left(\eta^{-1}(\cdot), M\right)$ satisfies $\tilde{\rho}<$ id by (6). This shows Definition $6-($ ii). So $V$ defined in (7) is a global finite-time Lyapunov function for system (1).

Let us briefly discuss Assumption 12. First of all, by definition, any GES system is bounded by a $\mathcal{K} \mathcal{L}$-function $\beta(r, t)=C \mu^{t} r$ with $C \geq 1, \mu \in[0,1)$, see (3). So we can find an $M \in \mathbb{N}$ such that $C \mu^{M}<1$, which immediately yields the following proposition.

Proposition 14. If system (1) is GES, then Assumption 12 is satisfied.

This now implies that for a GES system (1) any scaled norm is a global finite-time Lyapunov function.

Corollary 15. If system (1) is GES, then for any function $\eta \in \mathcal{K}_{\infty}$ the function $V: \mathbb{R}^{n} \rightarrow \mathbb{R}_{+}$ defined in (7) is a global finite-time Lyapunov function for this system.

Proof. Proposition 14 implies that Assumption 12 holds, so Theorem 13 applies and the result follows.

If we assume system (1) to be GAS only, then (6) does not have to hold globally, i.e., for all $r>0$. But we can at least show that Assumption 12 is satisfied in a semi-global practical sense.

Lemma 16. Assume system (1) is GAS. Then for any $0<a<b<\infty$ there exists an $M \in \mathbb{N}$, such that (6) holds for all $r \in[a, b]$. 
Proof. Let $\beta \in \mathcal{K} \mathcal{L}$ satisfy (2) for system (1). Using [9, Lemma 4.3] there exist $\mathcal{K}_{\infty}$-functions $\sigma_{1}, \sigma_{2} \in \mathcal{K}_{\infty}$ such that $\beta(r, t) \leq \sigma_{1}\left(\sigma_{2}(r) e^{-t}\right)$ holds for all $r, t \geq 0$. Define for any $r>0$

$$
M(r):=\min \left\{M \in \mathbb{N}: \sigma_{1}\left(\sigma_{2}(r) e^{-M}\right)<r\right\} .
$$

Note that $M(r)$ is well-defined for any $r>0$. Let $0<a<b<\infty$ be given and define $\bar{M}:=$ $\sup \{M(r): r \in[a, b]\}$. We have to show that $\bar{M}<\infty$, then for all $r \in[a, b]$ it holds

$$
\beta(r, \bar{M}) \leq \sigma_{1}\left(\sigma_{2}(r) e^{-\bar{M}}\right) \leq \sigma_{1}\left(\sigma_{2}(r) e^{-M(r)}\right)<r .
$$

So assume to the contrary that $\bar{M}=\infty$. Then there exists a sequence $\left\{r_{l}\right\}_{l \in \mathbb{N}} \in[a, b]$ such that $\left\{M\left(r_{l}\right)\right\}_{l \in \mathbb{N}} \rightarrow \infty$ for $l \rightarrow \infty$. Since $[a, b] \subset \mathbb{R}_{+}$is compact, we can without loss of generality assume that the sequence $\left\{r_{l}\right\}_{l \in \mathbb{N}}$ is convergent to a point $r^{*} \in[a, b]$, else take a convergent subsequence. But this means that in any open neighborhood $U$ around $r^{*}$ there exist infinitely many $r_{i} \in U$ with $M\left(r_{i}\right)$ pairwise distinct. On the other hand $M\left(r^{*}\right)$ is well-defined, and, by continuity, there exists an open neighborhood $\tilde{U}$ around $r^{*}$ with $M(\tilde{r}) \leq M\left(r^{*}\right)$ for all $\tilde{r} \in \tilde{U}$. But this contradicts the unboundedness of the sequence $\left\{M\left(r_{i}\right)\right\}$, where $r_{i} \in U \subset \tilde{U}$. So $\bar{M}<\infty$.

This now implies that for any GAS system of the form (1), any scaled norm is a semi-global practical finite-time Lyapunov function as defined next.

Definition 17. A function $V: \mathbb{R}^{n} \rightarrow \mathbb{R}_{+}$is a practical finite-time Lyapunov function for system

(1) if there exist $0<a<b<\infty$

(i) there exist $\alpha_{1}, \alpha_{2} \in \mathcal{K}_{\infty}$ such that for all $\xi \in \mathbb{R}^{n}$

$$
\alpha_{1}(\|\xi\|) \leq V(\xi) \leq \alpha_{2}(\|\xi\|)
$$

(ii) there exists a finite $M \in \mathbb{N}$ and a $\mathcal{K}$-function $\rho<$ id such that for all $\xi \in B_{[a, b]}:=\left\{\xi \in \mathbb{R}^{n}\right.$ : $\|\xi\| \in[a, b]\}$

$$
V(x(M, \xi)) \leq \rho(V(\xi))
$$

If for any $0<a<b<\infty$ there exists an $M \in \mathbb{N}$ such that item (ii) holds, then $V$ is a called a semi-global practical finite-time Lyapunov function.

In this case the function $V: \mathbb{R}^{n} \rightarrow \mathbb{R}_{+}$defined in (7) with arbitrary $\eta \in \mathcal{K}_{\infty}$ ensures practical asymptotic stability on $[a, b]$, i.e., any solution $x(k, \xi)$ with $\xi \in B_{[a, b]}$ converges to the set $B_{[0, a]}$. Note that enlarging $M \in \mathbb{N}$ enlarges the set $[a, b]$ on which practical asymptotic stability may be ensured. In particular, if $b=\infty$ the function $V$ is a global practical finite-time Lyapunov function.

Corollary 18. If system (1) is GAS, then for any function $\eta \in \mathcal{K}_{\infty}$ the function $V: \mathbb{R}^{n} \rightarrow \mathbb{R}_{+}$ defined in (7) is a semi-global practical finite-time Lyapunov function for this system, and the system is semi-globally practically stable.

Proof. Lemma 16 implies that for any $0<a<b<\infty$ there exists an $M \in \mathbb{N}$ such that (6) holds for all $r \in[a, b]$. Hence the $\mathcal{K}$-function $\tilde{\rho}$ in the proof of Theorem 13 satisfies $\tilde{\rho}(r)<r$ for all $r \in[\eta(a), \eta(b)]$. So for all $\xi \in B_{[a, b]}$ the function $V$ defined in (7) is strictly decreasing at least any $M$ steps. Since 0 is the only equilibrium point of $V$ this shows that the set $[0, a]$ is attractive for all initial states $\xi \in B_{[a, b]}$. Since $a>0$ and $b<\infty$ in Lemma 16 can be chosen arbitrarily the system is semi-globally practically stable.

\subsection{Construction of a global Lyapunov function from a global finite-time Lyapunov function}

In this section we construct a global Lyapunov function for system (1) from the knowledge of a global finite-time Lyapunov function $V: \mathbb{R}^{n} \rightarrow \mathbb{R}_{+}$as defined in Definition 6 . We want to 
emphasize that this construction does not depend on the particular choice of the global finite-time Lyapunov function in (7), but rather holds in general. The construction is as follows:

$$
W(\xi):=\sum_{j=0}^{M-1} V(x(j, \xi)),
$$

and yields the following result.

Theorem 19. (Construction of a global Lyapunov function I) If $V: \mathbb{R}^{n} \rightarrow \mathbb{R}_{+}$is a global finite-time Lyapunov function for system (1) with $M \in \mathbb{N}$ satisfying Definition 6-(ii), then $W: \mathbb{R}^{n} \rightarrow \mathbb{R}_{+}$defined in (8) is a global Lyapunov function for system (1).

Proof. For the global finite-time Lyapunov function $V$ let $\alpha_{1}, \alpha_{2}, \rho, M$ satisfy Definition 6 . In the remainder of the proof we will construct $\tilde{\alpha}_{1}, \tilde{\alpha}_{2}, \tilde{\rho}$ such that they satisfy Definition 4 for the Lyapunov function candidate $W$ defined in (8). Assume that $\alpha_{1}, \alpha_{2} \in \mathcal{K}_{\infty}$ are given such that $\alpha_{1}(\|\xi\|) \leq V(\xi) \leq \alpha_{2}(\|\xi\|)$. Let $\tilde{\alpha}_{1}=\alpha_{1}$, then by definition of $W$ we have $\tilde{\alpha}_{1}(\|\xi\|) \leq V(\xi) \leq W(\xi)$. Since $V$ is a global finite-time Lyapunov function we have for all $k \in \mathbb{N}$

$$
\begin{aligned}
\|x(k, \xi)\| & \leq \alpha_{1}^{-1}(V(x(k, \xi))) \leq \max _{j \in \mathbb{N}} \alpha_{1}^{-1}(V(x(j, \xi))) \\
& \leq \max _{j \in\{0, \ldots, M-1\}} \alpha_{1}^{-1}(V(x(j, \xi))) \\
& \left.\leq \max _{j \in\{0, \ldots, M-1\}} \alpha_{1}^{-1} \circ \alpha_{2} \circ \omega^{j}(\|\xi\|)\right)=: \sigma(\|\xi\|)
\end{aligned}
$$

for $\sigma:=\max _{j \in\{0, \ldots, M-1\}} \alpha_{1}^{-1} \circ \alpha_{2} \circ \omega^{j} \in \mathcal{K}_{\infty}$. Then

$$
\begin{aligned}
W(\xi) & \leq M \max _{i \in\{0, \ldots, M-1\}} V(x(i, \xi)) \\
& \leq M \max _{i \in\{0, \ldots, M-1\}} \alpha_{2}(\|x(i, \xi)\|) \leq M \alpha_{2} \circ \sigma(\|\xi\|) .
\end{aligned}
$$

Define $\tilde{\alpha}_{2}=M \operatorname{id} \circ \alpha_{2} \circ \sigma$, then we have

$$
\tilde{\alpha}_{1}(\|\xi\|) \leq W(\xi) \leq \tilde{\alpha}_{2}(\|\xi\|) .
$$

To show the decay of $W$ note that for any $\xi \in \mathbb{R}^{n}$

$$
\begin{aligned}
W(x(1, \xi)) & =\sum_{j=1}^{M} V(x(j, \xi)) \\
& \leq \sum_{j=1}^{M-1} V(x(j, \xi))+\rho(V(\xi)) \\
& =W(\xi)-(\mathrm{id}-\rho)(V(\xi)) \\
& \leq W(\xi)-(\mathrm{id}-\rho)\left(\alpha_{1}(\|\xi\|)\right) \\
& \leq\left(\mathrm{id}-(\mathrm{id}-\rho) \circ \tilde{\alpha}_{1} \circ \tilde{\alpha}_{2}^{-1}\right)(W(\xi)) \\
& =: \tilde{\rho}(W(\xi)) .
\end{aligned}
$$

By similar arguments as in Remark 5 we see that $0<\tilde{\rho}<$ id. This shows that $W$ is a global Lyapunov function for system (1) by Definition 4 .

An alternative construction to the one given in (8) is given by

$$
W(\xi):=\max _{j \in\{0, \ldots, M-1\}} \rho^{j / M}(V(x(M-1-j, \xi))) .
$$

With this construction we obtain an analogue to Theorem 19. 
Theorem 20. (Construction of a global Lyapunov function II) If $V: \mathbb{R}^{n} \rightarrow \mathbb{R}_{+}$is a global finite-time Lyapunov function for system (1) with $M \in \mathbb{N}$ satisfying Definition 6-(ii), then $W: \mathbb{R}^{n} \rightarrow \mathbb{R}_{+}$defined in (9) is a global Lyapunov function for system (1).

Proof. The proof follows the lines of the proof of Theorem 19 and is therefore omitted. Note that we have $W(x(M, \xi)) \leq \tilde{\rho}(W(\xi))$ with $\tilde{\rho}:=\rho^{1 / M}$.

\subsection{Converse Lyapunov theorems}

Now we can state the main results of this work as a corollary of Theorem 13 and Theorem 19 resp. Theorem 20.

Corollary 21. (Converse Lyapunov theorem I) Let $M \in \mathbb{N}$ satisfy Assumption 12. Then for any function $\eta \in \mathcal{K}_{\infty}$ the function $W: \mathbb{R}^{n} \rightarrow \mathbb{R}_{+}$defined by

$$
W(\xi):=\sum_{j=0}^{M-1} \eta(\|x(j, \xi)\|)
$$

for all $\xi \in \mathbb{R}^{n}$ is a global Lyapunov function for system (1).

Corollary 22. (Converse Lyapunov theorem II) Let $M \in \mathbb{N}$ satisfy Assumption 12. Then for any function $\eta \in \mathcal{K}_{\infty}$ the function $W: \mathbb{R}^{n} \rightarrow \mathbb{R}_{+}$defined by

$$
W(\xi):=\max _{j \in\{0, \ldots, M-1\}} \rho^{j / M} \circ \eta(\|x(M-1-j, \xi)\|)
$$

for all $\xi \in \mathbb{R}^{n}$ is a global Lyapunov function for system (1).

Remark 23. The main difference of the construction of the Lyapunov functions in Corollary 21 and 22 in contrast to the constructions of Lyapunov functions in other converse theorems, is that we use a finite sum of solutions instead of an infinite sum [3], and we use the maximum over a finite set instead of the supremum over all solutions and all times $[8,5]$.

Since any GES system satisfies Assumption 12 (see Proposition 14), we obtain the following converse Lyapunov theorem for GES systems of the form (1).

Corollary 24. If system (1) is GES then for any $\eta \in \mathcal{K}_{\infty}$ the function $W$ defined in (10) resp. (11) is a global Lyapunov function for system (1) for a suitable $M \in \mathbb{N}$.

Proof. Since the system is GES, there exist $C \geq 1$, and $\mu \in[0,1)$ such that $\|x(k, \xi)\| \leq C \mu^{k}\|\xi\|$. Take $M \in \mathbb{N}$ such that $C \mu^{M}<1$, then Assumption 12 holds. Then by Corollary 21 resp. Corollary 22 the function $W$ defined in (10) resp. (11) is a global Lyapunov function for system (1).

Combining Corollary 18 and Theorem 19 resp. Theorem 20 we immediately obtain a semi-global practical Lyapunov function.

Corollary 25. If system (1) is GAS, then the function $W$ defined in (10) resp. (11), with suitably large $M \in \mathbb{N}$, is a semi-global practical Lyapunov function for system (1).

In Section 5 we will present several classes of systems for which the results presented in this section have particular consequences of relevance. For more general systems the following procedure enables us to check the stability at least semi-globally practically. Note that this procedure may not be successful for any system.

Remark 26 (Methodology). Given a system of the form (1) we compute $x(k, \xi)$ for $k \in \mathbb{N}$ and compute $^{2}$ an $M \in \mathbb{N}$ with $\|x(M, \xi)\|<\|\xi\|$ for $\xi \in B_{[a, b]}$ with $0<a<b<\infty$. In this case the function $V: \mathbb{R}^{n} \rightarrow \mathbb{R}_{+}$defined in (7) with arbitrary $\eta \in \mathcal{K}_{\infty}$ is a practical finite-time Lyapunov function, and it in particular ensures practical asymptotic stability on $[a, b]$, see Corollary 18.

\footnotetext{
${ }^{2}$ The existence is guaranteed by Lemma 16 .
} 
Defining $W: \mathbb{R}^{n} \rightarrow \mathbb{R}_{+}$by (10) or (11) then gives a practical Lyapunov function for the overall system (1). If $a=0$ then we obtain a Lyapunov function and if, additionally, $b=\infty$, then the Lyapunov function is even a global one.

Computation of a suitable $M$ can be done by iteratively checking the condition $\|x(M, \xi)\|<\|\xi\|$ while increasing the value of $M$. At least, when the dynamics is GES, there always exists an $M$ large enough for which the condition holds. The difficulty of checking this condition is depending on the particular map $G$. Systematic methods for obtaining an $M$ for linear and conewise linear maps are given in the next section.

\section{Relevant implications}

This section exploits the developed converse theorems to obtain relevant implications for several classes of dynamical systems.

We start with a result that is already known (see e.g. [3]), but with the construction of the global Lyapunov function $W$ in (10) in Corollary 21 (respectively (11) in Corollary 22) the proposed approach yields a much simpler proof.

Proposition 27. Let system (1) satisfy Assumption 12. If $G$ in (1) is continuous then there exists a continuous global Lyapunov function for system (1).

Proof. From Corollary 21 we directly obtain the global Lyapunov function

$$
W(\xi):=\sum_{j=0}^{M-1} \eta\left(\left\|G^{j}(\xi)\right\|\right)
$$

from (10). Alternatively, from Corollary 22 we directly obtain the global Lyapunov function

$$
W(\xi):=\max _{j \in\{0, \ldots, M-1\}} \rho^{j / M} \circ \eta\left(\left\|G^{M-1-j}(\xi)\right\|\right)
$$

from (11). In both cases the composition of continuous functions $(\eta, \rho, G)$ yields a continuous function, implying that $W$ is a continuous global Lyapunov function.

Note that using smoothing techniques (see e.g. [5, Sec. 3]) the function $W$ from (10) resp. (11) can be made smooth.

Remark 28. If $G: \mathbb{R}^{n} \rightarrow \mathbb{R}^{n}$ is a polynomial function satisfying Assumption 12 then there exists a polynomial global Lyapunov function for system (1). This follows by taking $\eta(s):=s^{2}$ in the global Lyapunov function in (10), and noticing that the sum and the composition of polynomial functions yields a polynomial function.

The following result can be obtained for another relevant type of map $G$, which was considered in [20] and [21].

Proposition 29. Let $G$ in (1) be a positively homogeneous of degree one map ${ }^{3}$ and let system (1) be GAS. Then there exists a global Lyapunov function for system (1) that is positively homogeneous of degree one.

Proof. If the map $G$ is positively homogeneous of degree one and system (1) is GAS, it holds by Corollary V.3 in [21] that system (1) is GES, i.e., GAS (or equivalently $\mathcal{K} \mathcal{L}$-stability as defined in this work) is equivalent with GES for homogeneous dynamics. Then, by Corollary 24 the function $\eta$ in (12) can be taken as a positively homogeneous function of degree one. The proof is completed by observing that vector norms are positively homogeneous functions of degree one as well and that the composition of a finite number of such functions remains a positively homogeneous function of degree one.

\footnotetext{
${ }^{3}$ I.e., for any $\xi \in \mathbb{R}^{n}, G(c \xi)=c G(\xi)$ for any $c>0$.
} 
In the next two sections we consider the cases of conewise linear and linear dynamical systems in more detail.

\subsection{Conewise linear dynamical systems}

Next, we focus on a relevant class of hybrid systems, i.e., conewise linear systems, see, e.g., the survey [22], for which the results in this paper turn out to be quite useful. In [17] it was shown that conewise linear Lyapunov functions are sufficient for establishing GES of conewise linear systems and that such functions can be computed by linear programming. See also [23], which focuses on the discrete-time setting. The open question that remains to be answered is whether conewise linear Lyapunov functions are also necessary for GES conewise linear systems. In what follows we make use of the results of this paper to answer this question affirmatively, within the discrete-time setting.

To this end, firstly, a formal characterization of conewise linear dynamics is given.

Definition 30. A non-empty set $\mathcal{C} \subset \mathbb{R}^{n}$ is a cone if and only if

(i) $\operatorname{int} \mathcal{C} \neq \emptyset$, where $\operatorname{int} \mathcal{C}$ denotes the interior of the set $C$;

(ii) $\mathcal{C} \cap-\mathcal{C}=\{0\}$;

(iii) $c \xi \in \mathcal{C}$ for any $\xi \in \mathcal{C}$ and $c \in \mathbb{R}_{+}$;

(iv) $\lambda \xi_{1}+(1-\lambda) \xi_{2} \in \mathcal{C}$ for any $\xi_{1}, \xi_{2} \in \mathcal{C}$ and $\lambda \in(0,1)$

A finite set of cones $\left\{\mathcal{C}_{i} \subset \mathbb{R}^{n}\right\}_{i \in\{1, \ldots, l\}}$ defines an l-conic partition of $\mathbb{R}^{n}$, if the following holds for $i, j \in\{1, \ldots, l\}$

(i) $\bigcup_{i} \mathcal{C}_{i}=\mathbb{R}^{n}$

(ii) $\operatorname{int} \mathcal{C}_{i} \cap \operatorname{int} \mathcal{C}_{j}=\emptyset$ for all $i \neq j$.

Next, consider the class of conewise linear dynamics, i.e.

$$
G(x):=A_{i} x \quad \text { if } \quad x \in \mathcal{C}_{i} ; \quad i \in\{1, \ldots, N\},
$$

where $N \in \mathbb{N}, A_{i} \in \mathbb{R}^{n \times n}$, and the finite set of cones $\left\{\mathcal{C}_{i}\right\}_{i \in\{1, \ldots, N\}}$ defines an $N$-conic partition of $\mathbb{R}^{n}$. Observe that $G$ of $(13)$ satisfies Assumption 1 with $\omega(s):=\max _{i \in\{1, \ldots, N\}}\left\|A_{i}\right\| s$. For any solution $x(k, \xi)$ of the conewise linear system

$$
x(k+1)=A_{i} x(k) \quad \text { if } \quad x(k) \in \mathcal{C}_{i}
$$

with $x(0)=\xi \in \mathbb{R}^{n}$, we associate the $k$-tuple $\left(j_{1}, \ldots, j_{k}\right), j_{i} \in\{1, \ldots, N\}$, if $x(l, \xi) \in \mathcal{C}_{j_{l+1}}$. Unifying these sets we have

$$
\begin{aligned}
\mathcal{I}_{k} & :=\left\{\left(j_{1}, \ldots, j_{k}\right) \in\{1, \ldots, N\}^{k}:\right. \\
& \left.A_{j_{k}}\left\{\ldots\left\{A_{j_{2}}\left\{A_{j_{1}} \mathcal{C}_{j_{1}} \cap \mathcal{C}_{j_{2}}\right\} \cap \mathcal{C}_{j_{3}}\right\} \cap \ldots \cap \mathcal{C}_{j_{k}}\right\} \neq \emptyset\right\} .
\end{aligned}
$$

Note that the set $\mathcal{I}_{k}$ can be computed by basic operations (image under $A_{j_{i}}$ and intersection) involving cones and/or via reachability graphs. These operations can be performed efficiently for the case of polyhedral cones, i.e., cones represented as the convex hull of a finite number of rays, see. e.g., [24].

Furthermore, we define the set

$$
\mathcal{A}_{k}:=\left\{\left[\prod_{i=0}^{k-1} A_{j_{k-i}}\right]:\left(j_{1}, \ldots, j_{k}\right) \in \mathcal{I}_{k}\right\},
$$

where $\left[\prod_{i=0}^{k-1} A_{j_{k-i}}\right]:=A_{j_{k}} A_{j_{k-1}} \ldots A_{j_{1}}$, and for $k=0$ this product is defined as the identity matrix $I$. 
The following theorem states that GES of a conewise linear system (14) is equivalent to the existence of a conewise linear Lyapunov function. Notice also that conewise linear maps are positively homogeneous maps of degree one and, as such, GAS as defined in this work, or equivalently $\mathcal{K} \mathcal{L}$ stability, is equivalent with GES by Corollary V.3 of [21]. As such, without any loss of generality we can state the following result in terms of GES.

Theorem 31. The conewise linear system (14) is GES if and only if it admits a global conewise linear Lyapunov function.

Proof. In [23, Thorem 4.6] it is shown that the existence of a conewise linear Lyapunov function implies GAS of system (14), and hence even GES. So in this proof we consider the converse statement.

Let system (14) be GES. Then Corollary 15 implies that the function $V: \mathbb{R}^{n} \rightarrow \mathbb{R}_{+}$defined by $V(\xi):=\|\xi\|_{1}$ is a global finite-time Lyapunov for system (14). Furthermore, let $M \in \mathbb{N}$ satisfy Definition 6-(ii). Hence, applying Corollary 24 we see that the function $W: \mathbb{R}^{n} \rightarrow \mathbb{R}_{+}$defined by $W(\xi):=\sum_{k=0}^{M-1}\|x(k, \xi)\|_{1}$ is a global Lyapunov function for system (14).

For any $\xi \in \mathbb{R}^{n}$ let $\iota:=\left(j_{1}, \ldots, j_{M}\right) \in \mathcal{I}_{M}$ be associated to the solution $x(\cdot, \xi)$. Note that the number $\# \mathcal{I}_{M}$ of non-identical $M$-tuples in $\mathcal{I}_{M}$ is at most $N^{M}$. Then for any $\iota=\left(j_{1}, \ldots, j_{M}\right) \in \mathcal{I}_{M}$ we define the set

$$
\begin{aligned}
& \mathcal{D}_{\iota}:=\left\{\xi \in \mathcal{C}_{j_{1}}: x(k, \xi) \in \mathcal{C}_{j_{k+1}} \forall k \in\{1, \ldots, M-1\}\right\}= \\
& \left\{\xi \in \mathcal{C}_{j_{1}}:\left[\prod_{j=0}^{k-1} A_{j_{k-j}}\right] \xi \in \mathcal{C}_{j_{k+1}} \forall k \in\{1, \ldots, M-1\}\right\} .
\end{aligned}
$$

Consider the union of the sets $D_{\iota}, \bigcup_{\iota \in \mathcal{I}_{M}} \mathcal{D}_{\iota}$. Note that this union defines a partition of $\mathbb{R}^{n}$, since solutions $x(k, \xi)$ are defined for any time $k \in \mathbb{N}$ and any initial point $\xi \in \mathbb{R}^{n}$. Since the sets $\mathcal{C}_{i}$ are cones, it is easy to see that the sets $\mathcal{D}_{\iota}$ are cones. Furthermore, int $\mathcal{D}_{\iota} \cap \operatorname{int} \mathcal{D}_{\kappa}=\emptyset$ for any $\iota \neq \kappa$, because the cones $\mathcal{C}_{i}$ define an $N$-conic partition. Thus, $\bigcup_{\iota \in \mathcal{I}_{M}} \mathcal{D}_{\iota}$ defines a conic partition of $\mathbb{R}^{n}$.

For any cone $\mathcal{D}_{\iota}$ there exists a matrix $P_{\iota} \in \mathbb{R}^{p \times n}$ with $p \geq n$ such that

$$
\sum_{k=0}^{M-1}\left\|\left[\prod_{i=0}^{k-1} A_{j_{k-i}}\right] \xi\right\|_{1}=\left\|P_{\iota} \xi\right\|_{1}
$$

for all $\xi \in \mathcal{D}_{\iota}$. This matrix $P_{\iota} \in \mathbb{R}^{p \times n}$ can be chosen as

$$
P_{\iota}=\left(\prod_{i=0}^{M-2} A_{j_{M-1-i}} ; \ldots ; A_{j_{2}} A_{j_{1}} ; A_{j_{1}} ; I\right)
$$

where $I$ denotes the identity matrix. In particular, $p=M n$. Then the global Lyapunov function takes the explicit form

$$
\begin{aligned}
W(\xi) & =\sum_{k=0}^{M-1}\|x(k, \xi)\|_{1}=\sum_{k=0}^{M-1}\left\|\left[\prod_{i=0}^{k-1} A_{j_{k-i}}\right] \xi\right\|_{1} \\
& =\left\|P_{\iota} \xi\right\|_{1} \text { if } \xi \in \mathcal{D}_{\iota} .
\end{aligned}
$$

Since weighted 1-norms are conewise linear functions, see, for example, [11], and $\bigcup_{\iota \in \mathcal{I}_{M}} \mathcal{D}_{\iota}$ defines a conic partition of $\mathbb{R}^{n}$ we obtain that $W$ is a conewise linear function, which concludes the proof.

Remark 32. The proof of Theorem 31 is constructive as it yields a global conewise linear Lyapunov function $W$. In this proof we use the sum formulation (10) of Corollary 24 for the 1-norm to construct the Lyapunov function $W$. An alternative is to use the max formulation (11) of Corollary 24 for the infinity norm as follows.

Since we have a conewise linear system, the function $\rho \in \mathcal{K}$ in (11), corresponding to the global finite-time Lyapunov function $V(\xi)=\|\xi\|_{\infty}$ in Definition 6, can be chosen linear. Thus $\rho(s)=c s$ 
with $c \in(0,1)$. Using the same conic partition $\mathbb{R}^{n}=\bigcup_{\iota \in \mathcal{I}_{M}} \mathcal{D}_{\iota}$ as in the proof of Theorem 31, we have for $\xi \in \mathcal{D}_{\iota}$,

$$
\begin{aligned}
W(\xi) & =\max _{k \in\{0, \ldots, M-1\}} \rho^{k / M}\left(\|x(k, \xi)\|_{\infty}\right) \\
& =\max _{k \in\{0, \ldots, M-1\}} \rho^{k / M}\left(\left\|\left[\prod_{i=0}^{k-1} A_{j_{k-i}}\right] \xi\right\|_{\infty}\right) \\
& =\max _{k \in\{0, \ldots, M-1\}}\left\|c^{k / M}\left[\prod_{i=0}^{k-1} A_{j_{k-i}}\right] \xi\right\|_{\infty} \\
& =\left\|P_{\iota} \xi\right\|_{\infty}
\end{aligned}
$$

with

$$
P_{\iota}=\left(c^{\frac{M-1}{M}} \prod_{i=0}^{M-2} A_{j_{M-1-i}} ; \ldots ; c^{\frac{2}{M}} A_{j_{2}} A_{j_{1}} ; c^{\frac{1}{M}} A_{j_{1}} ; I\right),
$$

where $P_{\iota} \in \mathbb{R}^{n M \times n}$. Hence $W$ is a global conewise linear Lyapunov function for system (14). This is the infinity norm analogue to the 1-norm construction in Theorem 31.

The above results, besides establishing non-conservatism of conewise linear Lyapunov functions for stability analysis of conewise linear systems, provide an explicit construction of such Lyapunov functions. The construction depends on finding an admissible value of the positive integer $M$, related to the finite-time Lyapunov condition, which hinges on computing the set $\mathcal{A}_{k}$. In the case of polytopic cones, reachability analysis ${ }^{4}$ for conewise linear systems can be performed efficiently, which yields $\mathcal{A}_{k}$. Hence, finding admissible values of $M$ for conewise linear dynamics with polytopic conic partitions is tractable whenever $\mathrm{P}=\mathrm{NP}$ for the corresponding stability analysis problem [16]. Otherwise, no other stability analysis method can provide a tractable test.

Example 33. To illustrate the above results, consider a discontinuous dynamics that corresponds to (13), but with $N=4, A_{1}=A_{3}=\left(\begin{array}{cc}0.197 & -0.241 \\ 1.845 & 1.703\end{array}\right), A_{2}=A_{4}=\left(\begin{array}{cc}-0.638 & -0.823 \\ 0.175 & 0.538\end{array}\right)$, where $A_{1}=A_{3}$ is unstable. The corresponding conic partition is defined by $\left\{\mathcal{C}_{i}\right\}_{i \in\{1, \ldots, 4\}}$ with $\mathcal{C}_{i}=\left\{x \in \mathbb{R}^{2}\right.$ : $\left.E_{i} x \geq 0\right\}$ for all $i \in\{1, \ldots, 4\}$, and where $E_{1}=-E_{3}=\left(\begin{array}{cc}1 & -1 \\ 1 & 1\end{array}\right), E_{2}=-E_{4}=\left(\begin{array}{cc}1 & -1 \\ -1 & -1\end{array}\right)$. To make use of the results developed in this paper, we first indicate that the function $V(x)=\|x\|_{1}$ is a finite-time Lyapunov function with $M=18$, which was established by computing $\left\|\prod_{i=1}^{k} A_{j_{i}}\right\|_{1}$ for all $\left(j_{1}, \ldots, j_{k}\right) \in \mathcal{A}_{k}$ for $k=1, \ldots, 18$. Hence, the function $W(\xi):=\sum_{k=0}^{M-1}\|x(k, \xi)\|_{1}$ is a global Lyapunov function for this system. In Figure 1 we show a contour plot of the constructed non-convex conewise linear Lyapunov function. Note that $W$ is conewise with respect to a conic partition $\mathcal{D}_{\iota}$, which is fines than that given by $\mathcal{C}_{1}, \ldots, \mathcal{C}_{4}$. This follows from the trajectorywise definition of $W$. The effect can be seen in Figure 1, where discontinuities of $W$ occur along rays.

\footnotetext{
${ }^{4}$ As implied by the results in [16], this may be an NP-hard problem for conewise linear systems.
} 


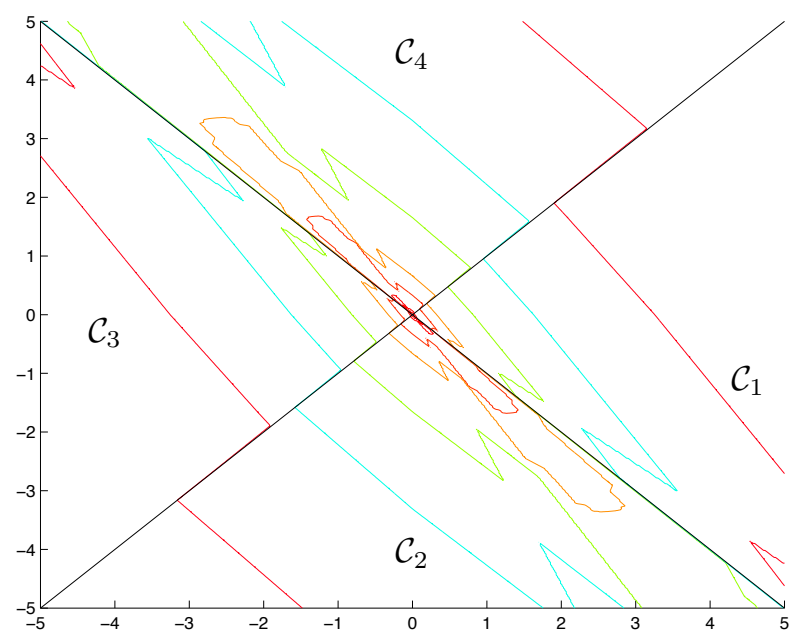

Figure 1: Contour plot of the Lyapunov function $W(\xi):=\sum_{i=0}^{17}\|x(k, \xi)\|_{1}$ for Example 33 .

Example 34. Consider Example 6.1 in [22]. In this example the author showed the global exponential stability of a conewise linear system, where two out of three of the linear dynamics ${ }^{5} \operatorname{are}^{-}$ unstable. Similarly as done in the previous example, one can compute $M=4$ for the infinity norm case, and $M=3$ for the 1-norm case, for which the corresponding finite-time Lyapunov function condition is met. Hence, the function $V(\xi):=\|\xi\|_{1}$ is a global finite-time Lyapunov function by Theorem 13, and the function $W(\xi):=\sum_{i=0}^{2}\|x(k, \xi)\|_{1}$ is a global Lyapunov function for this system. A contour plot showing the sublevel sets of $W$ is given in Figure 2.

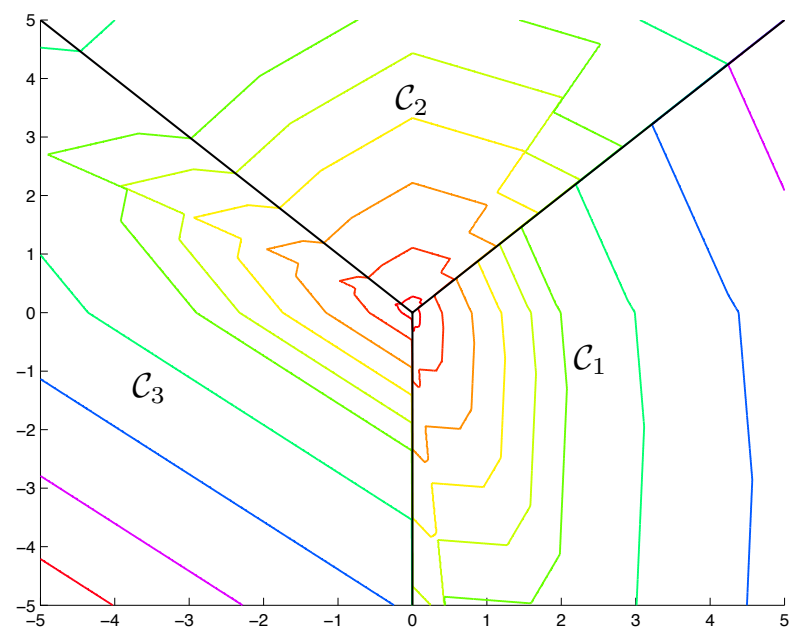

Figure 2: A contour plot of the Lyapunov function $W(\xi):=\sum_{i=0}^{2}\|x(k, \xi)\|_{1}$ in Example 34 .

\section{$5.2 \quad$ Linear dynamical systems}

Interestingly, if the conewise linear dynamics reduces to the standard linear dynamics, with the convention that this dynamics is valid in $\mathbb{R}^{n}$, Theorem 31 implies that GES is equivalent to the

\footnotetext{
${ }^{5}$ Please note the typo in [22]: The matrices $A_{2}$ and $A_{3}$ have to be interchanged.
} 
existence of a global polyhedral ${ }^{6}$ Lyapunov function of the form $W(\xi):=\|P \xi\|_{1, \infty}$. In this case the Lyapunov weight matrix $P \in \mathbb{R}^{p \times n}$ with $p \geq n$ is not square in general, but of full column rank. Polyhedral Lyapunov functions [10], [11] are in fact convex conewise linear functions, which can be expressed as the maximum over a finite number of linear functions (see also [25] for further insights).

The above observation is formally stated next.

Corollary 35. A linear system

$$
x(k+1)=A x(k)
$$

with $k \in \mathbb{N}$, and $A \in \mathbb{R}^{n \times n}$ is $G E S$ if and only if there exists a global polyhedral Lyapunov function of the form $W(\xi)=\|P \xi\|_{1}$ with $P \in \mathbb{R}^{p \times n}, p \geq n$.

In particular, if $M \in \mathbb{N}$ satisfies $C \mu^{M}<1$ with $C \geq 1, \mu \in[0,1)$ satisfying (3) and (2), then the number of rows of $P$ can always be chosen as $p=M n$.

Proof. It remains to show that GES of the linear system (15) implies the existence of a matrix $P \in$ $\mathbb{R}^{p \times n}$ such that $W(\xi)=\|P \xi\|_{1}$ is a Lyapunov function. First note that by Corollary 24, GES implies that there exists an $M \in \mathbb{N}$ such that the function $W(\xi):=\sum_{k=0}^{M-1}\|x(k, \xi)\|_{1}=\sum_{k=0}^{M-1}\left\|A^{k} \xi\right\|_{1}$ is a global Lyapunov function. In particular, $M$ satisfies $C \mu^{M}<1$, where $C \geq 1, \mu \in[0,1)$ stem from (3) and (2). Exploiting the fact

$$
\sum_{k=0}^{M-1}\left\|A^{k} \xi\right\|_{1}=\|P \xi\|_{1}
$$

with

$$
P:=\left(A^{M-1} ; A^{M-2} ; \ldots ; A ; I\right)
$$

we obtain that $W(\xi)=\|P \xi\|_{1}$ is a global Lyapunov function for the linear system (15). Note that $P$ has full column rank by the identity matrix in the last row block. In particular, this implies that for a linear GES system (15) there always exists a Lyapunov function $W(\xi)=\|P \xi\|_{1}$, where $P$ has $p=M n$ number of rows.

Remark 36. Corollary 35 constructs a suitable global Lyapunov function $W$ as a weighted 1-norm. Following Remark 32 it is easy to see that $W(\xi):=\|P \xi\|_{\infty}$, i.e., a weighted infinity norm, is a global Lyapunov function as well. In this case, however, the matrix $P$ is defined as

$$
P=\left(\left(c^{1 / M} A\right)^{M-1} ; \ldots ; c^{2 / M} A^{2} ; c^{1 / M} A ; I\right),
$$

where $c \in(0,1)$ and $\rho(s):=c s$ satisfies Definition 6-(ii) for the global finite-time Lyapunov function $V(\xi)=\|\xi\|_{\infty}$.

Remark 37. In [10], [11] and, among several other works, [25], existence of a polyhedral Lyapunov function $W(\xi)=\|P \xi\|_{1, \infty}$ with $P \in \mathbb{R}^{p \times n}$ is established for GES linear systems. However, the proofs therein are rather complex and not constructive. In particular, no estimate of an upper bound on the number of rows $p$ of the Lyapunov weight matrix $P$ is given. This is in fact one of the non-trivial, open problems in construction of polyhedral Lyapunov functions for linear systems, see, e.g., [26], [27]. Corollary 35 solves this problem by explicitly giving an admissible value of $p$ for the 1-norm case, while an admissible value of $p$ for the infinity norm case is given in Remark 36 . In both cases $p=M n$, where $M$ is derived from the corresponding global finite-time Lyapunov function $V(\xi)=\|\xi\|_{1, \infty}$.

Based on the above results and insights, we are in a position to provide a systematic procedure for constructing polyhedral Lyapunov functions for linear systems that is applicable in state spaces of high dimension. Note that this is attained without employing a (Jordan) decomposition of the $A$ matrix or any further assumptions on the eigenvalues of $A$, as done in existing works on this topic, see, e.g., [25] and the references therein. To this end, in view of the proof of Corollary 24, it is

\footnotetext{
${ }^{6}$ I.e., the sublevel sets of such a function are convex polyhedra with zero in their interior.
} 
possible to obtain an admissible value for $M$ analytically, for linear systems. The procedure is as follows. Consider the matrix inequalities

$$
\begin{aligned}
\rho(A)^{2} P-A^{\top} P A & \succeq 0, \\
c_{2} I_{n} \succeq P & \succeq I_{n},
\end{aligned}
$$

where $c_{2} \geq 1$ and $\rho(A)$ denotes the spectral radius of $A$ (the dynamics of the linear system). There exists a matrix $P$ that satisfies the LMIs (16) if and only if the linear system is GES, see, e.g., [4]. A matrix $P$ that satisfies (16) can be found by solving the LMIs (16) while minimizing $c_{2}$. Thus, it follows from standard Lyapunov arguments, see, e.g., [3], that the GES property $\|x(k, \xi)\|_{2} \leq C \mu^{k}\|\xi\|_{2}$ holds with $\mu:=\rho(A), C:=\sqrt{c_{2}}$ for the Euclidian norm $\|\cdot\|_{2}$. Hence, it follows from the condition $C \mu^{M}<1$ that any $M \in \mathbb{N}$ such that $M>\log _{\mu}\left(\frac{1}{C}\right)$ is admissible in the sense that it provides a valid finite-time Lyapunov function. Note that for the 1-norm and the infinity norm case, we have to choose $C:=\sqrt{n c_{2}}$, which follows by the norm equivalences in $\mathbb{R}^{n}$. The polyhedral Lyapunov function is then directly obtained from Corollary 35 or Remark 36 , respectively.

An alternative to the LMI approach to computing a suitable $M \in \mathbb{N}$ was obtained in [21]. Take any norm $\|\cdot\|$. Then it holds $\|x(k, \xi)\|=\left\|A^{k} \xi\right\| \leq\left\|A^{k}\right\|\|\xi\|$. Hence, we have $M:=\min \{k \in \mathbb{N}$ : $\left.\left\|A^{k}\right\|<1\right\}$. As we can see in the following examples, this second approach yields smaller values for $M \in \mathbb{N}$ compared to the LMI approach.

Example 38. To illustrate the results for linear dynamics, consider system (15) with $A=$ $\left(\begin{array}{cc}1 & 0.4 \\ -0.2 & 0.9\end{array}\right)$. We construct Lyapunov functions both for the 1-norm case and for the infinity norm case. (i) In the 1-norm case we obtain $M=74$ by the LMI approach (16), whereas the second approach for computing $M \in \mathbb{N}$ yields $\|x(k, \xi)\|_{1} \leq\left\|A^{k}\right\|_{1}\|\xi\|_{1}$, and $\left\|A^{11}\right\|_{1}<1$. Hence for $M=11$, the function $W_{1}(\xi)=\left\|P_{1} \xi\right\|_{1}$ is a conewise linear Lyapunov function for system (15), where $P_{1} \in \mathbb{R}^{22 \times 2}$ can be computed in a straightforward manner by Corollary 35 . In Figure 3 we provide a contour and surface plot of the Lyapunov function $W_{1}$.

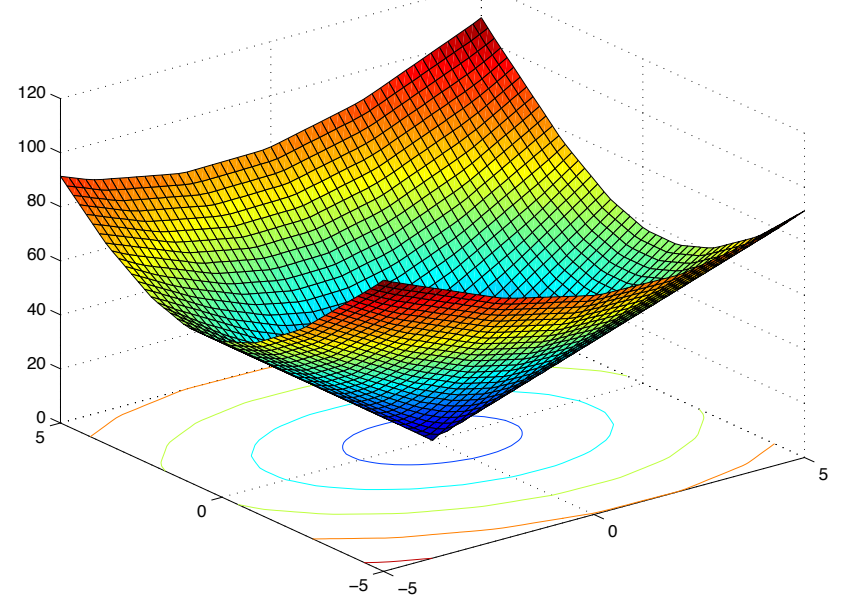

Figure 3: Surface plot of the Lyapunov function $W_{1}$ of Example 38.

(ii) In the infinity norm case, again, we obtain $M=74$ by the LMI approach (16). Alternatively, $\left\|A^{11}\right\|_{\infty}<1$ implies that $V(\xi)=\|\xi\|_{\infty}$ is a global finite-time Lyapunov function for the linear system (15) satisfying $V(x(11, \xi)) \leq\left\|A^{11}\right\|_{\infty} V(\xi)$. Hence for $M=11$, the function $W_{\infty}(\xi)=$ $\left\|P_{\infty} \xi\right\|_{\infty}$ is a conewise linear Lyapunov function for system (15), where $P_{\infty} \in \mathbb{R}^{22 \times 2}$ can be computed in a straightforward manner by Remark 36, where we took $c=0.91$. 
Example 39. To illustrate the applicability of the developed methods in high dimension state spaces, consider the linear system (15) with

$$
A=\left[\begin{array}{cccccccccc}
0 & -0.3 & 0.1 & -0.1 & -0.3 & 0 & -0.1 & -0.1 & 0 & -0.4 \\
-0.4 & 0.4 & -0.3 & 0.1 & -0.2 & 0.1 & -0.4 & 0 & -0.2 & 0.4 \\
-0.4 & -0.1 & 0.1 & 0.2 & -0.3 & -0.3 & 0 & 0.2 & 0.3 & 0.3 \\
-0.4 & -0.3 & 0.2 & -0.1 & -0.1 & -0.1 & 0 & -0.1 & -0.2 & 0.4 \\
0.3 & 0.2 & 0.4 & 0.2 & -0.2 & -0.4 & -0.3 & -0.4 & 0.1 & 0.2 \\
-0.4 & 0.4 & 0.2 & 0.3 & -0.2 & -0.1 & 0.4 & -0.2 & -0.3 & 0.4 \\
0 & 0.2 & 0.2 & 0 & 0.2 & -0.3 & -0.3 & -0.4 & 0.2 & -0.1 \\
0.4 & 0.3 & 0.4 & 0.3 & 0 & 0 & 0.4 & 0.3 & 0.4 & 0 \\
0.2 & 0 & -0.2 & -0.3 & -0.3 & 0 & 0 & 0 & 0.2 & 0.2 \\
0 & 0.1 & 0 & -0.1 & 0.4 & 0.3 & -0.2 & 0.3 & 0.4 & 0.3
\end{array}\right] .
$$

This systems is GES as the spectral radius of $A$ is 0.9544 , and hence, less than 1 . So we can compute the value $M \in \mathbb{N}$ by the LMI approach (16) as $M=39$. Using the alternative approach, by computing minimal $k \in \mathbb{N}$ satisfying $\left\|A^{k}\right\|<1$, we obtain for the 1 -norm case a value of $M_{1}=17$, and for the infinity-norm case a value of $M_{\infty}=20$. Thus, by Corollary 35, we obtain the linear Lyapunov function $W_{1}(\xi)=\left\|P_{1} \xi\right\|_{1}$, where the matrix $P_{1} \in \mathbb{R}^{170 \times 10}$ is given by Corollary 35 . Following Remark 36, we obtain the linear Lyapunov function $W_{\infty}(\xi)=\left\|P_{\infty} \xi\right\|_{\infty}$ with matrix $P_{\infty} \in \mathbb{R}^{200 \times 10}$.

\section{Conclusions}

This paper presented a novel converse Lyapunov theorem for discrete-time systems under a mild assumption. The developed theorem is constructive, as it provides an explicit Lyapunov function, whereas other converse Lyapunov theorems only show existence of a Lyapunov function. Moreover, we showed that for specific classes of systems, the developed converse theorem can be used to establish non-conservatism of a particular type of Lyapunov functions. Most notably, a proof that conewise linear Lyapunov functions are non-conservative for GES conewise linear systems was given and, as a by-product, a tractable construction of polyhedral Lyapunov functions for linear systems was attained.

\section{References}

[1] N. E. Barabanov, "Lyapunov indicator of discrete inclusions. I.," Autom. Remote Control, vol. 49, no. 2, pp. 152-157, 1988 .

[2] E. A. Barbašin and N. N. Krasovskiǔ, "On the existence of Lyapunov functions in the case of asymptotic stability in the large," Akad. Nauk SSSR. Prikl. Mat. Meh., vol. 18, pp. 345-350, 1954.

[3] Z.-P. Jiang and Y. Wang, "A converse Lyapunov theorem for discrete-time systems with disturbances," Systems Control Lett., vol. 45, no. 1, pp. 49-58, 2002.

[4] R. Kalman and J. Bertram, "Control system analysis and design via the second method of Lyapunov: (i) continuous-time systems (ii) discrete time systems," Automatic Control, IRE Transactions on, vol. 4, pp. 371-393, December 1959.

[5] C. M. Kellett and A. R. Teel, "On the robustness of $\mathcal{K} \mathcal{L}$-stability for difference inclusions: Smooth discrete-time Lyapunov functions," SIAM Journal on Control and Optimization, vol. 44, no. 3, pp. 777$800,2005$.

[6] I. G. Malkin, "On the reversibility of Lyapunov's theorem on asymptotic stability," Akad. Nauk SSSR. Prikl. Mat. Meh., vol. 18, pp. 129-138, 1954.

[7] J. L. Massera, "On Liapounoff's conditions of stability," Ann. of Math. (2), vol. 50, pp. 705-721, 1949.

[8] D. Nešić, A. R. Teel, and P. V. Kokotović, "Sufficient conditions for the stabilization of sampled-data nonlinear systems via discrete-time approximations," Systems and Control Letters, vol. 38, no. 4-5, pp. 259-270, 1999.

[9] A. R. Teel and L. Praly, "A smooth Lyapunov function from a class- $\mathcal{K} \mathcal{L}$ estimate involving two positive semidefinite functions," ESAIM - Control Optimization and Calculus of Variations, vol. 5, pp. 313$367,2000$.

[10] A. P. Molchanov and E. S. Pyatnitskii, "Criteria of asymptotic stability of differential and difference inclusions encountered in control theory," Systems 8 Control Letters, vol. 13, pp. 59-64, 1989. 
[11] H. Kiendl, J. Adamy, and P. Stelzner, "Vector norms as Lyapunov functions for linear systems," IEEE Transactions on Automatic Control, vol. 37, no. 6, pp. 839-842, 1992.

[12] S. F. Hafstein, An algorithm for constructing Lyapunov functions, vol. 8 of Electronic Journal of Differential Equations. Monograph. Texas State University-San Marcos, Department of Mathematics, San Marcos, TX, 2007.

[13] F. Camilli, L. Grüne, and F. Wirth, "Control Lyapunov functions and Zubov's method," SIAM J. Control Optim., vol. 47, no. 1, pp. 301-326, 2008.

[14] Z. Zhijun, "Converse Lyapunov theorems for nonautonomous discrete-time systems.," J. Math. Sci., New York, vol. 161, no. 2, pp. 337-343, 2009.

[15] D. Aeyels and J. Peuteman, "A new asymptotic stability criterion for nonlinear time-variant differential equations.," IEEE Trans. Autom. Control, vol. 43, no. 7, pp. 968-971, 1998.

[16] V. D. Blondel and J. N. Tsitsiklis, "Complexity of stability and controllability of elementary hybrid systems," Automatica J. IFAC, vol. 35, no. 3, pp. 479-489, 1999.

[17] M. Johansson, Piecewise linear control systems, vol. 284 of Lecture Notes in Control and Information Sciences. Springer, 2003.

[18] R. Geiselhart and F. R. Wirth, "Solving iterative functional equations for a class of piecewise linear $\mathcal{K}_{\infty}$-functions." submitted to J. Math. Anal. Appl., May 2013.

[19] M. Lazar, Model Predictive Control of Hybrid Systems: Stability and Robustness. PhD thesis, Eindhoven University of Technology, 2006.

[20] S. Emre Tuna, "Growth rate of switched homogeneous systems," Automatica, vol. 44, pp. 2857-2862, 2008.

[21] M. Lazar, S. V. Raković, A. I. Doban, and N. Athanasopoulos, "On stability analysis of discrete-time homogeneous dynamics," tech. rep., Eindhoven University of Technology, http://www.cs.ele.tue.nl/MLazar/TechReport03.pdf, 2013.

[22] Z. Sun, "Stability of piecewise linear systems revisited," Annual Reviews in Control, vol. 34, pp. 221$231,2010$.

[23] M. Lazar and A. Jokić, "On infinity norms as Lyapunov functions for piecewise affine systems," in Proc. of the ACM Conference Hybrid Systems: Computation and Control, (Stockholm, Sweden), pp. 131-139, 2010.

[24] G. M. Ziegler, Lectures on Polytopes, Updated Seventh Printing. Berlin New-York: Springer, 2007.

[25] M. Lazar, "On Infinity Norms as Lyapunov Functions: Alternative Necessary and Sufficient Conditions," in 49th IEEE Conference on Decision and Control, (Atlanta, GA, USA), pp. 5936-5942, December 15-17 2010.

[26] O. N. Bobyleva and E. S. Pyatnitskii, "Piecewise-linear Lyapunov functions and localization of spectra of stable matrices," Automation and Remote Control, vol. 62, no. 9, pp. 1417-1427, 2001. Translated from Avtomatika i Telemekhanika, No. 9, 2001, pp. 25-36.

[27] O. N. Bobyleva, "Piecewise-linear Lyapunov functions for linear stationary systems," Automation and Remote Control, vol. 63, no. 4, pp. 540-549, 2002. Translated from Avtomatika i Telemekhanika, No. 4, 2002, pp. 26-35. 